\title{
Adding contingency management intervention to vocational rehabilitation: Outcomes for dually diagnosed veterans
}

\author{
Charles E. Drebing, PhD; ${ }^{1 *}$ E. Alice Van Ormer, PhD; ${ }^{1}$ Lisa Mueller, MA; ${ }^{1}$ Marcie Hebert, PsyD; ${ }^{1}$ Walter E. \\ Penk, PhD, ABPP; ${ }^{1}$ Nancy M. Petry, PhD; ${ }^{2}$ Robert Rosenheck, MD; ${ }^{3}$ Bruce Rounsaville, MD $^{3}$ \\ ${ }^{1}$ Bedford Department of Veterans Affairs Medical Center, Bedford, MA; ${ }^{2}$ University of Connecticut Health Center, \\ Farmington, CT; ${ }^{3}$ Yale University School of Medicine, New Haven, CT
}

\begin{abstract}
In this random-assignment trial, we evaluated the efficacy of using a contingency management (CM) intervention to enhance job acquisition and tenure among participants of a vocational rehabilitation (VR) program. The CM intervention offered participants cash incentives up to $\$ 1,170$ for completing tasks related to sobriety and job search and maintenance. Participants were 100 veterans with comorbid psychiatric disorders and substance dependence who were randomly assigned either to VR only or VR + CM. Relative to participants in the VR-only group, those in the VR + CM group showed more intense job searches and transitioned to competitive employment faster and at higher rates. No significant difference was found in job tenure, though this may be due to the limited follow-up period. Abstinence rates were significantly better in the VR + CM group during the first 16 weeks of follow-up but not significantly different in subsequent follow-ups. No relationship was found between relapse and employment. These results suggest that rehabilitation outcomes may be enhanced by adding CM to current programming or by restructuring traditional work-for-pay contingencies to include direct financial rewards for achievement of clinical goals.
\end{abstract}

Key words: compensated work therapy, contingency management, dual diagnosis, employment, job search, job tenure, psychiatric rehabilitation, sobriety, vocational rehabilitation, work.

\section{INTRODUCTION}

Helping adults with psychiatric disabilities find and maintain employment is a national priority [1]. To address this issue, the Veterans Health Administration (VHA) has invested heavily in vocational rehabilitation (VR) programs, particularly Compensated Work Therapy (CWT), which is the Department of Veterans Affairs' (VA) largest clinical VR program. In less than 10 years, the number of veterans served annually by VA VR services has grown to more than 22,000 [2-3] and the amount of money paid annually to veterans through work-for-pay activities has grown to more than $\$ 34$ million [4]. The outcome for VA VR participants in terms of percentage employed in competitive jobs at discharge has increased almost every year since 1993 [5]. Despite this investment of effort and resources, the highest rate of competitive employment at discharge, achieved in fiscal year 2004, was 41.7 percent [5]. Dropout rates in some programs range as high as 70 percent, and as many as 40 percent of participants drop out before 4 weeks of participation [4]. Similar findings are noted among non-VA VR programs [6].

Abbreviations: $\mathrm{CM}=$ contingency management, $\mathrm{CWT}=\mathrm{Com}-$ pensated Work Therapy, DSM-IV = Diagnostic and Statistical Manual of Mental Disorders-Fourth Edition, HR = hazard rate, JSBI = Job Search Behaviors Index, VA = Department of Veterans Affairs, VHA = Veterans Health Administration, VR = vocational rehabilitation, VRS $=$ VR specialist.

* Address all correspondence to Charles E. Drebing, PhD; Bedford VA Medical Center, Psychology Service, 200 Springs Road, 116B, Bedford, MA 01730; 781-687-2462; fax: 781-687-2169. Email: Charles.Drebing@med.va.gov DOI: 10.1682/JRRD.2006.09.0123 
In prior work [4,7-8], we identified factors contributing to the modest outcomes for VA VR participants with psychiatric disorders: poor program compliance, early drop out, frequent substance abuse relapse, low rates of transition to competitive employment, inadequate support to sustain competitive employment, and financial and emotional disincentives for employment. Of veterans with psychiatric disorders who enter VR, 75 percent have comorbid substance use disorders [8], and the proportion of VR entrants who are dually diagnosed has grown steadily since 1995 [5]. Substance abuse among persons with severe mental illness substantially increases the risk of treatment noncompliance, premature termination, relapse, and rehospitalization [9-10]. For improved VA VR outcomes, the needs of clients with dual diagnoses must be more fully addressed [11-12].

If we look to the field of substance abuse treatment, we see that similar problems of poor compliance and modest outcomes have been addressed successfully by adding incentive-based contingency management (CM) techniques [13-14]. By using behavioral principles to enhance participants' incentives for completing treatment and meeting clinical goals, substance abuse programs have increased completion rates from 30 to 80 percent and sobriety rates from 15 to 70 percent [15-16]. The efficacy of these approaches is now well documented in more than 24 clinical trials [13-14,17-18].

Despite the empirical evidence of the efficacy of CM techniques, they have not yet been adapted widely for other clinical settings such as VR [14]. In two initial studies, vouchers were an effective reward for sustained abstinence in a work setting [19-21]. In two additional studies, activities related to returning to work were rewarded directly. Silverman and associates found that job-training attendance significantly improved with an incentive regimen that rewarded attendance with vouchers [22]. Petry and associates allowed participants in substance abuse treatment to select work-related goals for their CM program [23]. Fifty-nine percent of the sample chose at least one work-related goal to be rewarded. Success rates varied between behaviors (e.g., working on a résumé was successfully completed $50 \%$ of the time, identifying potential jobs $66 \%$, submitting job applications $87 \%$, and attending work $69 \%$ ), while all workrelated goals were successfully accomplished in 79 percent of the cases.

We recently published the results of an initial application of CM principals to enhance outcomes in a VA VR program [24]. In this study, 19 dually diagnosed veterans entering VR were randomly assigned to VR only $(n=8)$ or VR + CM $(n=11)$. Over the first 16 weeks of rehabilitation, those in the VR + CM group could earn cash incentives for meeting two sets of clinical goals: (1) abstaining from drugs and alcohol and (2) taking steps to obtain and maintain competitive employment. We offered an escalating schedule of incentives for negative urine and Breathalyzer screens biweekly over the 16 weeks and for steps toward obtaining and maintaining employment, such as creating a résumé, completing a job interview, and working at a competitive job. Primary outcome variables were (1) placement into competitive employment and the time (in days) to placement, (2) hours of paid work and earnings, (3) substance abuse, and (4) program retention.

Results documented that participants in the VR + CM condition fared better on all outcome measures relative to those in VR only. The VR + CM group, compared with the VR-only group, was more likely to have created a résumé ( $81 \%$ vs $13 \%$, respectively), have completed a job interview ( $81 \%$ vs $25 \%$, respectively), and be working in a competitive job (45\% vs $25 \%$, respectively). Also, a larger percentage of the VR + CM group remained abstinent during the study (64\% vs $25 \%$, respectively).

The current study built on this initial pilot study to determine whether a revised version of this CM intervention applied to a larger sample of VR participants could improve treatment outcomes in terms of the number of participants obtaining and maintaining their own jobs. The incentive structure is a revised version of the pilot study intervention [24]. The specific behavioral targets for the incentives were selected based on the "pathways to reemployment" model [25]. In the pilot study, the number and value of incentives offered for work goals were less than those offered for sobriety goals (\$270 of total incentives for work goals vs $\$ 736$ for sobriety goals). Although the incentive group in that study completed work goals more often than the comparison group, further room for improvement existed in terms of rate of placement into competitive employment for the incentive group. We therefore increased the number and total value of incentives available for work goals and extended the availability period for employment incentives.

In a random-assignment trial of VR versus $\mathrm{VR}+\mathrm{CM}$, we examined whether VR + CM produced better employment outcomes than VR only. The primary hypotheses were that participants in the VR + CM condition would 
more rapidly transition to competitive employment and that those in the VR + CM condition who transitioned to competitive employment would have greater job tenure. Secondary analyses evaluated whether the VR + CM intervention effectively helped participants meet "intermediate” goals related to enhanced job-search intensity and sustained sobriety.

\section{METHODS}

\section{Participants}

For this study, we recruited 100 dually diagnosed veterans from among veterans entering the CWT program at the Bedford VA Medical Center (Bedford, Massachusetts). "Dually diagnosed veterans" were defined as those meeting Diagnostic and Statistical Manual of Mental Disorders-Fourth Edition (DSM-IV) criteria for a current diagnosis of (1) schizophrenia, bipolar disorder, major depression, posttraumatic stress disorder, or other anxiety disorder and (2) current drug or alcohol dependence or abuse, as well as active substance use within 90 days of enrollment. Because prior studies suggest that $\mathrm{CM}$ approaches to increasing abstinence are most effective when a relatively small number of substances are targeted $[23,26]$, we required participants to have substance dependence or abuse for alcohol, cocaine, or opiates and targeted those substances in the CM intervention.

Participants also had to have the potential for return to competitive supported employment within 6 months, as evidenced by a history of at least some participation in competitive employment during the prior 3 years and acceptance of the stated goal of returning to competitive employment within 8 months. They had to be clinically stable, defined as having no suicidal or homicidal ideation in the prior 12 weeks and abstaining from drugs or alcohol for at least 1 week. Veterans who were older than 55, had a chronic medical problem that would make obtaining and sustaining a competitive job within 8 months unlikely, or did not intend to stay in VR for at least 4 months or live in the local region for 12 months were considered less likely to be seeking VR participation to gain competitive employment and were excluded [27]. Candidates enrolled in other research studies that would affect their participation were also excluded. Because of the complexity of the incentives, we sought to exclude veterans who would have difficulty understanding the CM program. We therefore excluded any veteran who had less than 10 years of formal education, had a history of significant head trauma (loss of consciousness for $>1$ hour) or another disorder resulting in significant cognitive impairment, or failed to pass a 10 -item quiz about the incentives.

Veterans meeting all other criteria were given an overview and a paper summary of the intervention. To screen for potential participants who would have difficulty comprehending the intervention, we then asked them to complete the 10 -item quiz covering the content of the intervention. All potential subjects correctly completed the quiz. Potential participants were then invited to sign informed consent at the time of CWT admission.

A total of 101 veterans signed consent, completed the baseline evaluation, and completed random assignment. One participant was found to be ineligible for VA services because of a dishonorable discharge and so was excluded from the study. Of the remaining 100 participants (Table 1), the majority were non-Hispanic white, middle-aged males who had at least 12 years of formal education. Most had affective disorders (major depression $=79 \%$, bipolar disorder I or II $=21 \%$ ) or anxiety disorders (posttraumatic stress disorder $=53 \%$, other anxiety disorder $=50 \%$ ). Nine percent had a psychotic disorder. All met criteria for dependence on at least one of the three target substances, with 88 percent dependent on alcohol, 43 percent on cocaine, 26 percent on opiates, 29 percent on cannabis, 13 percent on sedatives, 5 percent on stimulants, and 3 percent on hallucinogens. Most were polysubstance dependent, with 33 percent of the sample dependent on two or more substances and 30 percent on three or more substances. Regarding employment, the average length of unemployment was 16.2 months and 46 percent stated that they lost their last job primarily because of substance abuse or psychiatric problems. On the Meaning of Work scale [28], most of the sample ranked work as very important, with 28 percent rating work as more important than all other life domains (family, religion, community, leisure) and 44 percent rating it only below family in importance. Financially, most were in substantial debt that outweighed their financial reserves, 26 percent were receiving some form of disability income, and 61 percent were receiving some form of public assistance.

Two subjects, both assigned to the VR-only condition, dropped out of the study during week 7 of the 16-week follow-up period. The follow-up rate was 94 percent for the 3-month follow-up, 90 percent for the 6-month followup, and 88 percent for the 9-month follow-up. 
JRRD, Volume 44, Number 6, 2007

Table 1.

Demographics and baseline variables of participants with comorbid psychiatric and substance use disorders who completed vocational rehabilitation (VR) only $(n=50)$ or VR plus contingency management (CM) $(n=50)$.

\begin{tabular}{|c|c|c|c|c|c|}
\hline Variable & Total Sample & VR Only & $\mathbf{V R}+\mathbf{C M}$ & $t$-Test $/ \chi^{2}$ & $p$-Value \\
\hline Ethnicity, $n(\%)$ & & & & 2.11 & 0.64 \\
\hline Hispanic & $5(5)$ & $2(4)$ & $3(6)$ & & \\
\hline Non-Hispanic & $95(95)$ & $48(96)$ & 47 (94) & & \\
\hline Race, $n(\%)$ & & & & 2.00 & 0.57 \\
\hline White & $78(78)$ & 39 (78) & 39 (78) & & \\
\hline African American & $20(20)$ & $10(20)$ & $10(20)$ & & \\
\hline Age, Mean \pm SD (yr) & $46.3 \pm 8.0$ & $47.2 \pm 6.7$ & $45.4 \pm 9.0$ & 1.11 & 0.27 \\
\hline Education, Mean \pm SD (yr) & $12.9 \pm 1.9$ & $12.7 \pm 1.7$ & $13.1 \pm 2.1$ & 1.25 & 0.21 \\
\hline Female, $n(\%)$ & $1(1)$ & $1(2)$ & $0(0)$ & 1.01 & 0.31 \\
\hline \multicolumn{6}{|l|}{ MOS SF-36: Role Limitation, Mean \pm SD } \\
\hline Medical & $71.2 \pm 23.3$ & $67.9 \pm 26.3$ & $74.6 \pm 19.3$ & 1.44 & 0.15 \\
\hline Meaning of Work Scale, Mean \pm SD & $6.0 \pm 1.2$ & $6.0 \pm 1.3$ & $6.0 \pm 1.1$ & 0.17 & 0.87 \\
\hline Baseline Job Search Behavior Index, Mean \pm SD & $11.3 \pm 8.4$ & $10.1 \pm 7.9$ & $12.6 \pm 8.9$ & 1.50 & 0.14 \\
\hline \multicolumn{6}{|l|}{ Financial Questionnaire, Mean \pm SD (\$) } \\
\hline Current Debt & $15,993 \pm 28,671$ & $14,521 \pm 29,423$ & $17,523 \pm 28,089$ & 0.52 & 0.60 \\
\hline Current Cash Reserves & $5,937 \pm 27,616$ & $6,927 \pm 31,082$ & $4,910 \pm 23,760$ & 0.36 & 0.72 \\
\hline Net Worth & $-10,054 \pm 40,435$ & $-7,595 \pm 44,095$ & $-12,614 \pm 36,517$ & 0.62 & 0.54 \\
\hline
\end{tabular}

\section{Design and Procedures}

Approximately 1,136 veterans were screened for the study. Detailed data were collected on the first 318 screened. Of those, 77 percent did not meet at least one screening criteria, 14 percent met criteria but did not enter, and 9 percent met criteria and entered the study. Of those who did not meet criteria, 20 percent did not have a psychiatric diagnosis, 9 percent did not have a substance abuse or dependence diagnosis, and 20 percent had a target substance use diagnosis but had been abstinent too long. Regarding employment, 18 percent of those not meeting the criteria had not worked competitively in 3 years, 15 percent were not seeking competitive employment within 8 months, and 9 percent were enrolled in treatment programs that limited their ability to participate fully in VR. Moreover, 8 percent were older than 55, 6 percent had recent suicidal or homicidal ideation, 2 percent had used substances in the prior 7 days, 2 percent had evidence of significant cognitive impairment, and 3 percent were already in another research study that would affect their participation. Of those who met criteria but declined participation, the stated reasons for declining included lacking confidence in their ability to obtain or maintain a competitive job (22\%), not wanting the obligation or investment of time required for participating (18\%), feeling that the intervention would overwhelm them (16\%), not wanting to complete job-search tasks (9\%), not wanting to undergo drug screening (4\%), and wanting to enter education instead of employment (13\%). Finally, 22 percent said they would consider participating but never returned and did not give a reason for not participating.

Those candidates who signed consent completed a baseline evaluation that included basic demographics, clinical assessment, and work history data collected with a demographics questionnaire and the CWT Work History Questionnaire [7]. Financial functioning was documented by a self-report questionnaire. Current psychiatric and substance use diagnoses were determined with the Structured Clinical Interview for DSM-IV [29]. A measure of rolelimitation due to either medical or emotional factors was administered (Medical Outcomes Study 36-item Short Form) [30]. Job-search intensity was assessed with an adapted form of the Job Search Behaviors Index (JSBI) [31], a brief 15-item checklist of job-search activities 
performed over the prior 3 months. The degree to which participants valued work was assessed with the Meaning of Work scale [28]. After the baseline evaluation, participants were randomly assigned to either VR only or VR + CM.

\section{Vocational Rehabilitation Only}

Both groups participated in VHA's CWT program and all CWT services were available to them. The Bedford CWT program is the largest in the country, with 700 unique veterans admitted each year and 250 participating on an average day [5,7]. Like other CWT programs, the Bedford CWT program is a multicomponent work-forpay VR program. Veterans are placed in structured work settings, usually in private companies in the metropolitan area, and compensated for their work. They are typically paid by CWT, which contracts with the company for their labor. While the veterans are working, the CWT staff help them negotiate and resolve difficulties on the job and prepare for obtaining their own competitive job. Like a growing number of CWT programs, the Bedford CWT program includes a supported employment component that helps participants maintain employment in their own competitive jobs through structured support and management [32-33]. Participants are encouraged to perform job-search tasks, abstain from drugs and/or alcohol, and obtain and then maintain competitive employment. The Bedford CWT program is similar in structure and outcome rates to other CWT programs around the country [5]. The mean hourly wage is $\$ 7.28$ [34]. For dually diagnosed participants, the average length of stay is 17 weeks and the transition to competitive employment for those who transition typically occurs after 22 weeks [7]. The only random-assignment evaluation of CWT found that participation was associated with reduced drug and alcohol abuse, fewer episodes of homelessness and incarceration, and protection from declined physical health relative to a control group [35].

\section{Vocational Rehabilitation Plus Contingency Management}

In addition to the usual CWT program just described, veterans assigned to the VR + CM group received additional incentives for taking steps toward obtaining and maintaining competitive employment and for abstinence from substance use.

\section{Incentives for Abstaining from Substance Use}

Of the possible clinical symptoms to target, we chose abstinence because of its direct tie to dropout [7] and demonstrated responsiveness to CM [14]. The "incentive" regimen for encouraging abstinence was modeled closely after approaches developed and validated by Higgins and colleagues $[15,26]$. A series of increasing cash incentives was offered for drug and alcohol screens negative for alcohol, cocaine, or opiates. We conducted onsite urine screening using the OnTrak TESTCUP 5 and the OnSite Alcohol Assay (Roche Diagnostics Inc, Indianapolis, Indiana). Screens were conducted twice weekly at unannounced times over the initial 16 weeks of the intervention. Screening results had to indicate no evidence of alcohol, cocaine, or opiate use to be considered negative or "clean." The incentive for the initial clean screen was worth $\$ 2.50$ and the value of each consecutive negative screen increased by $\$ 1.00$, such that the second consecutive negative screen was worth $\$ 3.50$, the third $\$ 4.50$, and so on. No payments were given if the screen was positive or if the participant did not produce a urine screen for any reason. Additionally, a positive screen or failure to provide a scheduled specimen reset the value of the incentives to their original level of $\$ 2.50$, from which they could increase again with consecutive negative screens. Four consecutive clean screens following a reset returned the incentive value to where it was before the reset. In total, participants could earn up to $\$ 560.00$ if all 32 samples tested clean during the 16 weeks.

\section{Incentives for Obtaining and Maintaining Competitive Employment}

Employment incentives were available in two phases. Phase I incentives targeted job-search tasks and were available for the first 16 weeks of the intervention. Phase II incentives targeted employment itself and were available for the first 32 weeks. To help participants meet the goal of obtaining a competitive job, we chose to reward the following job-search behaviors: conducting a job search, creating a résumé, completing a job application, networking, completing a job-relevant course, completing an informational interview, completing a mock interview, and completing a job interview. To help participants meet the goal of maintaining a competitive job, we chose to reward the following target behaviors: obtaining a competitive job and maintaining it for up to 4 months. Guidelines for these incentives were as follows:

1. As part of the study, participants were expected to participate in the Supported Employment track of the CWT program. Through this track, CWT staff were available to assist participants with each step in 
getting a job and support them in maintaining that job as long as the participants desired the support. If participants lost their job for any reason, CWT staff were available to help them find another job.

2. The job-search incentives were flexible to be more relevant to all participants. Participants could earn one incentive in each of the first 16 weeks by completing at least one target behavior. They could choose the behavior to complete, within some limits. For example, they could earn an unlimited number of incentives for completing job interviews but no more than one incentive for completing a résumé. Completing one target behavior during the first week resulted in payment of a $\$ 5$ incentive. The value of the subsequent incentives increased by $\$ 2$ for each consecutive week that the incentive was earned, such that the second incentive was worth $\$ 7$, the third $\$ 9$, and so on. Because we felt that consecutive weeks of job-search activities were less critical than consecutive weeks of sobriety, we did not reset the value of the job search incentives if the participant did not earn an incentive during a week.

3. Because participants could take several weeks to be ready to seek competitive employment, the availability of incentives for work was more flexible. Specifically, while the Phase I job-search incentives were available for the first 16 weeks of the study, the Phase II incentives were available for up to 32 weeks. Like maintaining sobriety, job-search activities were intermediate goals of the intervention and particularly important in the first 16 weeks of VR participation. Our pilot data suggest that job-search activities often are not completed in the first few months of CWT participation, reducing the likelihood of a timely transition to a competitive job. In contrast, the ultimate goals of obtaining and maintaining competitive employment typically occur only after the intermediate goals are accomplished. To ensure that most participants could achieve the Phase II target behaviors, these incentives were available for up to 32 weeks after the participant entered the study.

4. Participants could earn a job-search incentive for meeting with their CWT VR specialist (VRS) to learn or review how to conduct a job search. The VRS could show participants useful Web sites and other resources that list available jobs. These meetings were located at the VA and used computers available to participants for job searches. Participants needed to produce a printed computer job listing signed by their VRS to receive this incentive. To earn the incentive for a second job search, they needed to produce a printed computer job listing of jobs for which they were interested in applying or a written list of credible employers to whom they wished to apply. A "credible employer" was defined as any employer known to have an available job for which the participant may qualify. The listing had to have the date printed on it or be signed and dated by a computer laboratory or CWT staff member. For the third and fourth job searches, participants needed to produce a printed computer job listing and a fax machine printout showing that they had faxed their résumé to a potential employer. Computers and fax machines were available in the CWT facility. We did not limit the number of incentives that could be earned for completing job searches.

5. Participants could also earn a job-search incentive for having or creating a résumé that could be used to obtain a job. CWT staff were available to help participants develop a résumé. To earn an incentive, the résumé had to meet the following criteria, as judged by study staff:

a. Provided relevant information-name, address, telephone number, educational background, work history.

b. Was organized similar to résumés in résumé workbooks. Study or CWT staff members supplied participants with examples if necessary.

c. Looked professional-neatly printed on clean paper.

An incentive could be earned only once for creating a résumé.

6. Participants could earn a job-search incentive for conducting informational interviews at potential places of employment and providing written documentation of the interview. Up to four incentives could be earned for informational interviews.

7. Participants could earn a job-search incentive for networking. To earn this incentive, participants needed to write a description of who they talked with, when they talked with them, what they talked about, and how the discussion was related to a targeted job. Study staff had to agree that the contact was clearly linked to a targeted job for the incentive to be earned. Up to two incentives could be earned for networking. 
8. Participants could earn a job-search incentive for completing a mock job interview. CWT staff were available to conduct these mock interviews. To earn this incentive, participants needed a signed note from a VRS that stated that they had completed a mock interview. Up to two incentives could be earned for mock interviews.

9. Participants could also earn an incentive for applying for jobs. To earn the incentive, the participant had to send their résumé and/or completed job application to at least three potential employers and provide fax confirmation or other written documentation to study staff. We did not limit the number of incentives that could be earned for applying for jobs.

10. Participants could also earn an incentive for completing a real job interview. Participants needed to produce written evidence of an interview, similar to that used by the State Unemployment Office. We did not limit the number of incentives that could be earned for completing job interviews.

11. Because sustaining employment can be more difficult that obtaining employment and is the ultimate goal of VR, we designed the Phase II incentives to encourage consecutive weeks of employment. The value of the incentives increased with consecutive weeks of employment. Participants had to produce pay stubs or other written documentation of their work hours to receive the incentive. Participants could only earn incentives for the 16 weeks after beginning their first competitive job. During that time, they could change jobs or miss work, but they would not be paid incentives for times that they worked $<20$ hours a week.

12. The value of the incentive was $\$ 10$ for the first week of work, \$15 for the second week, \$20 for the third week, and \$25 for the fourth week. After 4 weeks of consecutive incentives, we diminished the frequency of incentives to facilitate the transition to naturally existing incentives. Participants could earn an incentive of $\$ 80$ for working a second consecutive month. This incentive was paid at the end of the month if participants provided pay stubs showing at least 20 hours of work a week for each of the 4 weeks in that month. The incentive value was $\$ 85$ for a third consecutive month of work and $\$ 90$ for a fourth consecutive month. If participants failed to work consecutive weeks, the value of the next incentive that could be earned was reset to $\$ 10$ for the first week of employment. Once participants began working, they could only earn incentives for working during the subsequent 16-week period.

13. To avoid creating a disincentive for participants to enter employment while the job-search incentives were available, participants entering employment during the first 16 weeks of the study also received incentive payments for completing Phase I tasks as long as they continued to be employed up to week 16.

14. Participants could only earn incentives if they were enrolled in CWT. If they were discharged from CWT for any reason, they could not earn incentives. If they did not maintain contact with staff members or stopped participating in their psychiatric care or any other care that VR requires, they could be discharged and unable to earn incentives.

15. All participants understood that if any question existed about whether an incentive had been earned, the research associate, in consultation with the project director, made the final determination.

In total, participants could earn up to $\$ 610$ if they successfully completed all work-related activities. Over the 36 weeks of the intervention, participants could earn incentives up to $\$ 1,170$. Participants were paid with either cash or a voucher for cash immediately redeemable at the hospital cashier, depending on whether going to the hospital cashier was reasonably convenient for the participant.

Because the incentive schedule is complex, we reviewed the intervention guidelines with all participants and gave three case examples. The participants then signed a summary agreement stating that they understood the intervention. They were given two copies of the signed contract, along with summary tables of the potential incentives and a summary of the incentives printed on a card to carry in their wallet. During meetings with study staff, participants were also encouraged to ask questions about the incentives.

Data collected during the first 16 weeks of study participation included measures of completed job-search tasks (assessed by weekly check-ins with the 15 JSBI items) and abstinence from alcohol, cocaine, and/or opiates (assessed by biweekly urine screens). Missing drugscreen data were considered positive, breaking the string of continuous abstinence. Missing income and employment data were assumed to reflect no income or no employment.

To ensure rapid and accurate onsite urine screening, we used the OnTrak TESTCUP 5 and OnSite Alcohol Assay. The OnTrak TESTCUP 5 provides a simultaneous screen 
for a panel of the five drugs that CWT participants most commonly abuse: cocaine, marijuana (THC), amphetamines, PCP, and morphine (300 ng/mL) [7]. Separate results were provided for each substance. Performance data supplied by the manufacturer indicated a $>99$ percent accuracy of the OnTrak TESTCUP 5 for all five target substances and a $>95$ percent precision [36]. Similar manufacturers' data indicated a 98 percent accuracy of the OnSite Alcohol Assay and good sensitivity and specificity [37]. During the study, all assays were conducted discreetly in a public restroom. The screenings provided results in $<3$ minutes, and staff gave immediate feedback and payment of incentives for those in the VR + CM group.

Participants in the VR-only group were paid \$5 per biweekly screen, for a total of $\$ 160$ over the initial 16 weeks, regardless of the test results. Clinicians and CWT staff could not access the urine-screen results for either group, and urine-screen results were not provided to participants in the VR-only group. The CWT program staff occasionally collected urine screens on their own, but the frequency of these was approximately once every 3 months except in unusual circumstances.

At the 3-, 6-, and 9-month follow-ups, we assessed job search and competitive employment variables using the CWT Work History and the JSBI. We assessed substance use using the time line follow-back method [38].

Since participants in the VR + CM group could earn more, we added an additional payment of $\$ 30$ for completing the final interview for those in the VR-only group. Participants in the VR-only group could receive up to $\$ 135$ for completing all data collection, and participants in the VR + CM group could receive up to \$105.

\section{Data Analysis}

We compared the two treatment groups on baseline characteristics that may be related to job-search and jobacquisition outcomes, including demographic variables, work history, financial resources and liabilities, disability income and other public support, limitations due to physical and mental illness, and job-search intensity, using $t$ tests for ordinal variables and chi-square tests for nominal variables. Some research hypotheses were also tested with $t$-tests for ordinal variables and chi-square tests for nominal variables.

All analyses were based on an intention-to-treat approach with $\alpha=0.05$. All tests of the primary hypotheses (A and B) were one-tailed. This meets Cohen's criteria for the appropriate use of a one-tailed test, given that the intervention is costly and would only be used if found to positively affect target variables [39]. Because the analyses for the remaining hypotheses (C and D) did not meet these criteria, these tests were two-tailed.

\section{Hypothesis A}

For hypothesis A, the primary outcome variable was number of weeks between intake and transition to competitive employment. Competitive employment was defined as the participant working at least 20 hours a week in an ongoing community-based job for which he or she was paid at least minimum wage. Because the goal of the intervention was sustained community-based employment, agency-contracted community employment, paid work activity at a business owned and run by a rehabilitation agency, and "casual" labor including day labor and temporary jobs were excluded from the outcome of competitive employment. We used a time-to-employment survival analysis with right-censored survival data to determine whether the hazard rate (HR) for transitioning to competitive employment was greater for the VR + CM group. The log-rank test compared time-to-employment between groups. We also compared the percentage of participants engaged in competitive employment during each month and the percentage that participated in competitive employment at any point during the study.

\section{Hypothesis $B$}

For hypothesis $\mathrm{B}$, the primary outcome variable was tenure of the first competitive job obtained, as indicated by the reported total number of days worked at that job. This variable was obtained through the CWT Work History, which was completed at the 3-, 6-, and 9-month follow-ups. Because job tenure was limited by the point in the follow-up when the job was obtained and jobs could potentially continue past the final follow-up, we again used a time-to-event analysis with right-censored survival data, with the target event being termination of the first job obtained. We used the log-rank test to compare time-to-end-of-first-job analyses between groups.

\section{Hypothesis $C$}

We evaluated hypothesis $C$ in two ways. First, we compared the two groups with respect to the total number of activities completed during each week and over the entire 16-week period when incentives were available and the frequency of each of the behaviors measured on the JSBI during the VR + CM intervention. Second, time- 
to-event survival analyses with use of right-censored survival data were used to determine whether the HR for three key job-search steps (creating a résumé, applying for a job, completing a job interview) during the 16 weeks that incentives were available was greater for the VR + CM group. Log-rank tests were again used to compare time-to-event analyses between groups.

\section{Hypothesis D}

Hypothesis D was evaluated in several ways. First, we used a time-to-relapse survival analysis with rightcensored survival data to determine whether the HR for relapse during the initial 16 weeks, when incentives for abstinence were available, was lower for the VR + CM group. An additional survival analysis determined whether the HR for relapse over the entire course of the study was lower for the VR + CM group. Log-rank tests were used to compare time-to-relapse between groups. Second, the percentages of participants in each group using targeted substances each week and over the entire 16-week period when incentives were available were compared by a series of chi-square tests. A simple $t$-test compared the longest period of sustained sobriety during the intervention. Substance use was measured with results from the biweekly screenings for weeks $1-16$, and self-report data from the time line follow-back between the 16-week point when the urine screens ended and the 9-month point.

\section{RESULTS}

Comparisons between the groups on baseline demographic characteristics indicated no significant differences at baseline (Table 1). No differences were found in diagnosis.

When the time-to-first-employment analysis was completed, participation in the VR + CM condition was associated with a significantly shorter time to entry into competitive employment $(\mathrm{HR}=1.88$, log-rank statistic $=$ 2.23, $p<0.05)$. As shown in Figure 1(a), 50 percent of the VR + CM group and 28 percent of the VR-only group had entered competitive employment by the end of the 9-month follow-up $\left(\chi^{2}(1, n=100)=5.09, p<0.05\right)$. We also compared the percentage of participants employed during each month (Figure 1(b)). While a greater percentage of the VR + CM group was employed at each month, the differences were statistically significant only for months 2, 7, and 9 .
When job tenure was examined, participation in VR + $\mathrm{CM}$ was not associated with statistically significantly longer tenure in competitive employment $(\mathrm{HR}=1.63$, logrank statistic $=1.18, p=0.29$ ). Note that this analysis was limited by the fact that only 39 study participants entered employment, 16 entering in the last 4 months of followup. Half of those who entered were still working in that position by the end of the follow-up period, further limiting information about tenure. Given that the incentive program rewarded job tenure of at least 4 months, we examined the subset of 18 participants (7 in the VR-only group and 11 in the VR + CM group) who entered work and had at least 4 months of follow-up data available for that position. No significant differences were noted in job tenure in this subgroup, as assessed with a simple $t$-test $(t(16)=0.14, p=0.90)$

Comparison between the groups with respect to jobsearch intensity fairly strongly supports hypothesis C. During the intervention, participants in the VR + CM group completed 39 percent more job-search tasks than those in the VR-only group (Table 2). When compared weekly during the intervention, the VR + CM group completed a greater number of tasks each week, though the differences were statistically significant only for weeks $1,6,7,9,11$, and 13 . When the frequency of completing specific job-search tasks was compared, the VR + CM group had a statistically significant higher completion rate on 5 of the 15 tasks (Table 2).

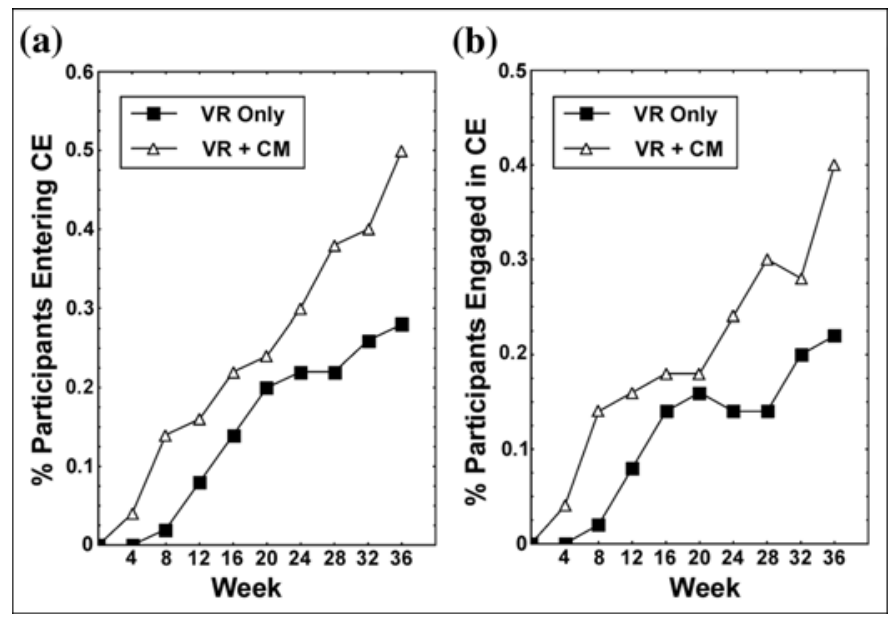

Figure 1.

Vocational rehabilitation (VR) only versus VR plus contingency management (CM) during 36 weeks of follow-up $(n=100,50$ each group): (a) time to competitive employment (CE) and (b) percentage engaged in CE. 
We used time to job-search task completion to determine whether the intervention also resulted in earlier completion of tasks. We examined three key tasks identified in prior studies [24]: résumé creation, first job application, and first job interview. As shown in Figure 2(a), 86 percent of the VR + CM group had completed a résumé by the end of the 16-week period compared with 68 percent of the VR-only group $(\mathrm{HR}=1.77$, log-rank statistic $=2.48$, $p<0.05)$. Similarly, 78 percent of the VR + CM group had submitted their first job application by week 16 as opposed to 50 percent of the VR-only group ( $\mathrm{HR}=2.13$, log-rank statistic $=2.61, p<0.01)($ Figure 2(b)). The difference in time to first job interview was smaller (Figure 2(c)), with 48 percent of the VR + CM group participating in at least one job interview by the end of the 16-week period compared with 32 percent of the VR-only group (HR $=1.60$, log-rank statistic $=0.94, p=0.35$ ). This difference was not statistically significant.

When time to first relapse was analyzed for weeks $1-$ 16, participation in the VR-only condition was associated with significantly shorter sobriety $(\mathrm{HR}=1.74$, log-rank statistic $=2.02, p<0.05)$. As shown in Figure 3, 50 percent of the VR + CM group had relapsed by week 16 in comparison with 72 percent of the VR-only group. If we examine the full 9-month follow-up period, the difference in relapse rate narrows, with 67 percent of the VR + CM group relapsing by the end of the 9-month follow-up compared with 75 percent of the VR-only group (HR $=1.25$, log-rank statistic $=1.34, p=0.18$ ). The difference between the groups is no longer statistically significant. The rates of abstinence for the VR + CM group were higher than those of the VR-only group for 14 of the 16 weeks, but the differences were statistically significant only during weeks 2 , 4, and 5 . When we compared the longest period of sustained sobriety during weeks $1-16$, the VR + CM group achieved a mean \pm SD of $11.8 \pm 4.7$ weeks versus $9.4 \pm$ 5.3 weeks for the VR-only group, a statistically significant difference $(t(98)=2.37, p<0.05)$.

We conducted additional analyses to examine the relationship between relapse and employment outcomes. Those participants who relapsed during weeks 1-16 were not significantly different from those who remained abstinent in terms of whether they entered competitive employment $\left(\chi^{2}(1, n=100)=0.13, p=0.90\right)$ or how quickly they entered employment $(\mathrm{HR}=1.03$, log-rank statistic $=0.01, p=0.91)$. Similarly, those participants who relapsed at any point during the study were not significantly different from those who remained abstinent in terms of whether they entered competitive employment $\left(\chi^{2}(1, n=100)=1.66, p=0.56\right)$ or how quickly they entered employment $(\mathrm{HR}=1.65$, log-rank statistic $=$ $0.36, p=0.23$ ).

Table 2 .

Job-search activity by group: Vocational rehabilitation (VR) only versus VR plus contingency management (CM) ( $n=100,50$ each group). Data shown as mean \pm standard deviation.

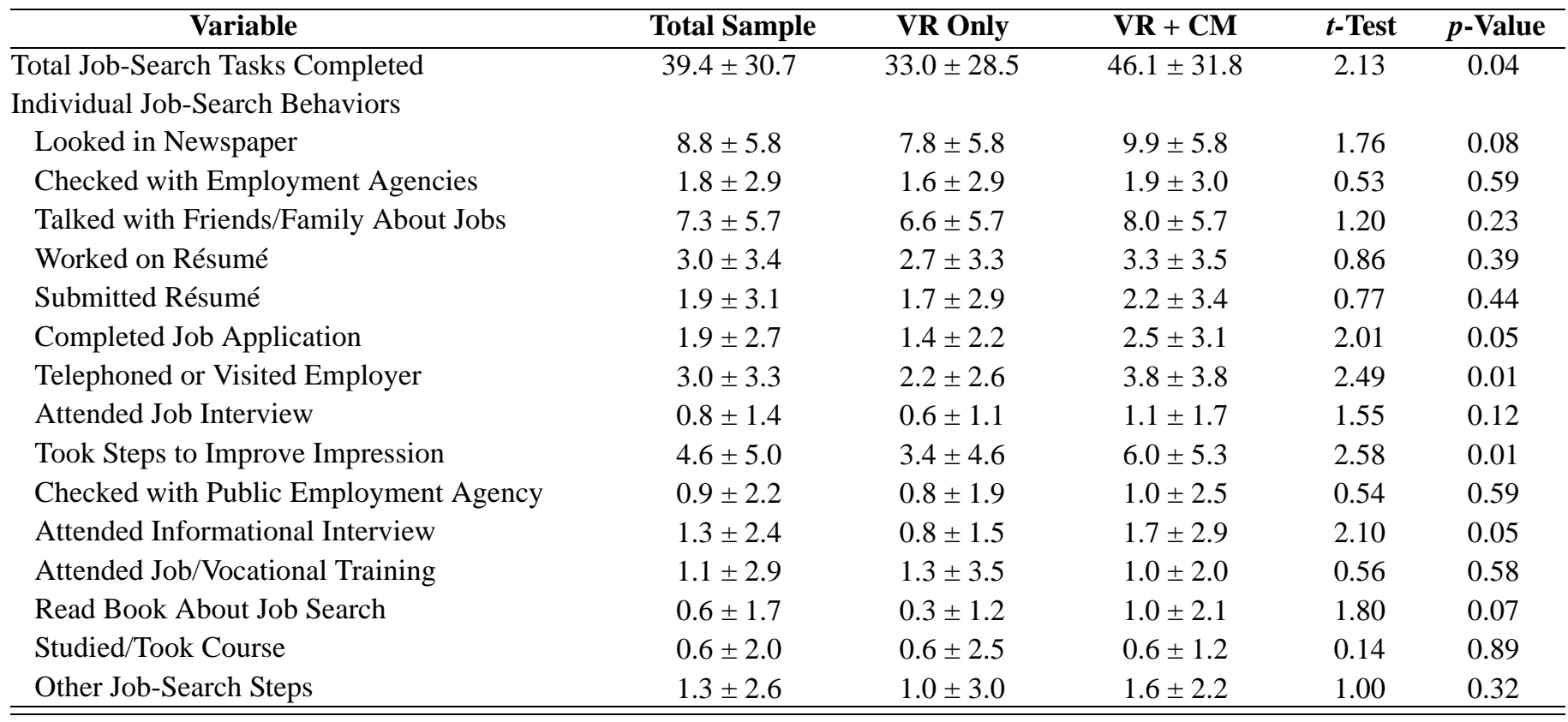




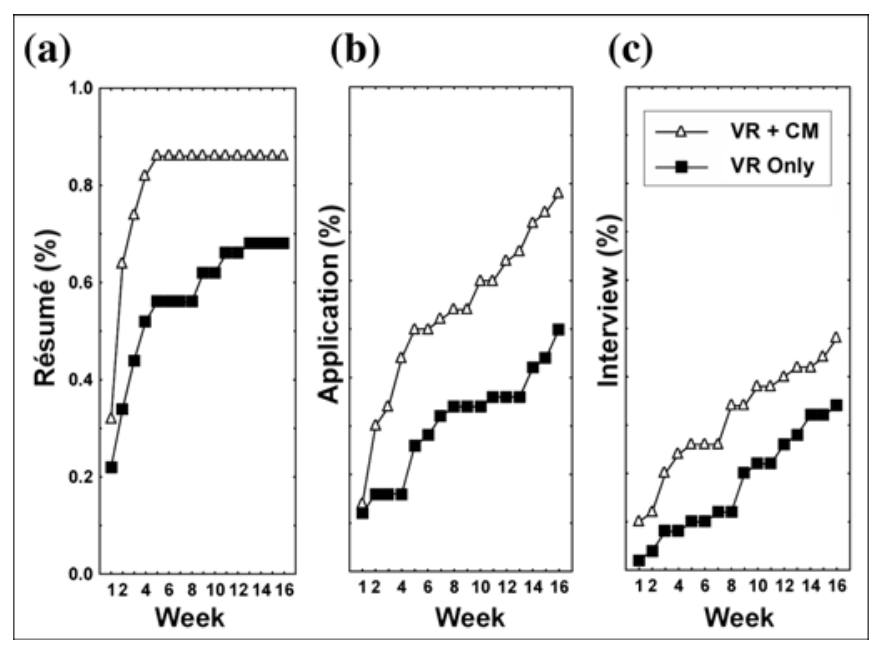

Figure 2.

Percentage of participants completing key job-search tasks during weeks 1-16; vocational rehabilitation (VR) only versus VR plus contingency management (CM): (a) résumé, (b) job application, and (c) job interview.

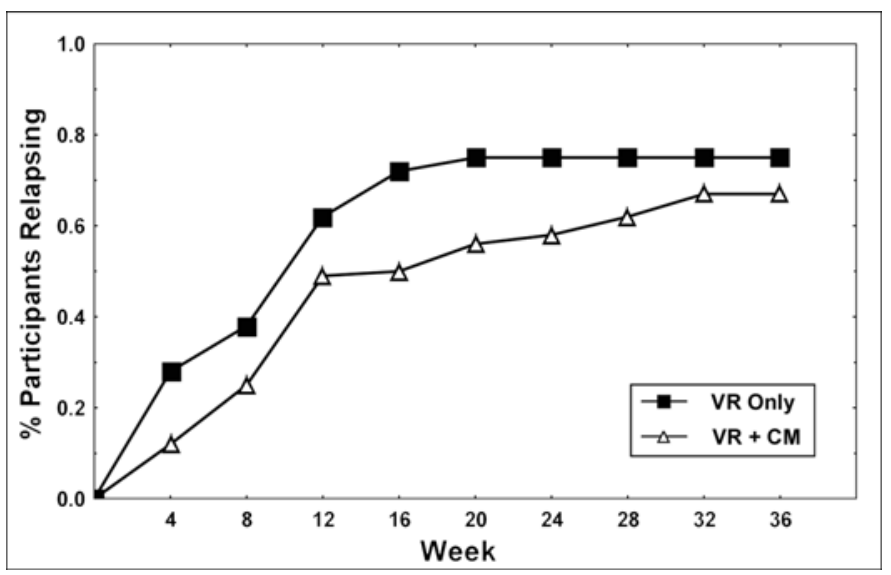

Figure 3.

Time to relapse during 9 months of follow-up; vocational rehabilitation (VR) only versus VR plus contingency management (CM). Note: Sobriety incentives were available weeks 1-16 only.

\section{DISCUSSION}

These results support the efficacy of adding CM intervention to VR to enhance entry into competitive employment, with entry rates being very similar to those achieved in the pilot study [24]. The VR + CM participants completed more job-search tasks and completed two of three target job-search tasks more quickly than the VR-only participants. Most importantly, they were more likely to transition to competitive employment and to transition more quickly than the VR-only participants. Less clear is whether the intervention had a lasting benefit for the intermediate goal of sustained sobriety. Also less clear is whether CM helped extend job tenure, though this may well be an artifact of the limited length of the follow-up and the number of participants who obtained jobs.

Regarding job-search activities, the incentives were associated not only with faster completion of two of three targeted tasks but also with more job-search activity overall. While the VR + CM participants were rewarded for completing up to one job task a week, on average they completed 3.1 tasks a week compared with the 2.2 completed by the VR-only participants. A comparison of individual items on the JSBI indicates that the VR + CM participants more frequently completed tasks that were not specifically rewarded than the VR-only participants. For example, they were more likely than the VR-only participants to report talking with friends, family, and others to get information about jobs ( $54 \%$ vs $43 \%$, respectively) and more likely to report improving their appearance to be more marketable to potential employers ( $40 \%$ vs $22 \%$, respectively). Interestingly, the differences in job-search intensity did not continue past the period in which the incentives were available, although this effect may have been a function of the fact that only the individuals least likely to be employed were still looking for employment by the time incentives for job-related activities were discontinued.

Of the targeted job-search tasks, the VR + CM group achieved a job interview more rapidly than the VR-only group, but the difference was not statistically significant. The percentage of the VR + CM group completing an interview (48\%) is lower than in the pilot study (82\%) [24]. Possibly, with the wider range of job-search behaviors that could earn an incentive in the revised intervention, participants did not choose the more anxiety-producing or more difficult-to-arrange tasks, like completing a job interview. In the pilot study, only four target behaviors could be rewarded and the reward for completing a job interview was among the highest in value, possibly resulting in the higher interview rate noted in that study. This change in interview rates is unfortunate, since completing a job interview is typically a crucial step in job acquisition and was closely related to employment entry in this study. The intervention should be further refined to ensure that, while adequate choice in job-search behaviors is available, 
that choice is structured so that the most critical behaviors are reinforced with the greatest magnitude to guarantee their completion.

The mixed substance abuse outcome of the CM intervention also differs from the findings of the pilot study and suggests that the relatively lower magnitude of incentives for sobriety in the current study may have reduced the impact on substance abuse, particularly after the incentives were no longer available. The finding that relapse was not significantly related to employment outcomes is somewhat surprising and likely reflects the complex relationship between relapse and employment for VR participants, at least during the initial period of VR participation. During the baseline evaluation, most study participants reported long histories of working competitively while using substances, suggesting that the negative impact of relapse on employment may not have been immediate enough to be documented in the followup period. Several participants reported that they actually moved to competitive employment after relapsing because they no longer felt confident that they could continue working in the CWT program without being "caught" and subsequently discharged. So in the short term, substance abuse may impel some VR participants to move to competitive jobs and may not be a large impediment to employment for others. This finding does raise questions about the necessity of incentives for abstinence in the intervention, particularly given their diminished effect in this protocol by the end of the follow-up period. It also suggests that the enhanced employment outcomes noted are most directly related to the incentives for employment and job search, as opposed to the incentives for sobriety.

Another important question is whether the VR + CM intervention results in more people entering competitive employment or simply encourages those who would enter to do so more rapidly. While the current study cannot fully answer this question, it is noteworthy that with a 9-month follow-up time, the difference in entry rates into employment continued to grow over the last 4 months of follow-up. If the VR + CM condition were simply encouraging a speedier entry into work by those who would simply enter later, one would expect the difference in the entry rates into employment to diminish over time. Future evaluation of CM interventions for work-related activities should examine effects over longer periods.

This study, along with the previous pilot study, documents the efficacy of a different application of CM tech- niques. Prior research focused almost exclusively on interventions that enhanced substance abuse treatment. While some studies focused on employment outcomes within substance abuse treatment settings, ours is the first to focus specifically on a VR setting. These results support the possibility of a wider range of clinical applications of CM techniques. CM techniques are often used to achieve repetition of targeted behaviors in order for participants to unlearn negative habits and increase learning of positive behavior patterns or increase compliance with treatment so that they receive a sufficient dose and achieve a clinical outcome. In this intervention, we identified key barriers to the positive goal of employment, identified behavioral targets that overcame those barriers, and then rewarded those targets. This different approach to $\mathrm{CM}$ incentives apparently facilitates the chain of behaviors required to successfully acquire employment. A similar approach would likely be well suited for improving outcomes for other rehabilitation services and goals.

Supported employment and motivational interviewing are two interventions that emphasize participants' intrinsic motivation to achieve rehabilitation goals like employment. Using extrinsic rewards to encourage employment in this $\mathrm{CM}$ intervention raises questions about whether CM has any effect on intrinsic motivation to work. Certainly, employment is a complex behavior governed by a wide range of intrinsic and extrinsic factors. Further study will be needed to better understand the effect of this CM intervention on this array of factors and to clarify whether combining interventions like motivational interviewing and CM would have an additive positive effect.

The current study has a number of limitations. First, the sample used in the study was clearly a select subgroup of VR participants and so findings cannot be generalized to the larger population of VR participants. A full 77 percent of candidates screened were excluded, and another 14 percent declined participation. Of note, many candidates who were excluded were actually insufficiently complex clinically. Still, many common VR participants have less clear "potential" for competitive employment in terms of poorer work history, lower commitment to employment, and greater clinical instability and so are not represented by the sample used here. Second, the intervention is fairly complex, raising the concern that potential problems with comprehension may limit its applicability in some VR settings. While study staff noted some evidence of difficulty 
understanding the incentives, it was fairly limited and easily addressed by the staff. Also worth noting is that only 4 percent of potential candidates were excluded because of cognitive limitations, and of the candidates who met the initial study criteria, all passed the 10 -item screening quiz. Third, the study is also limited by its reliance on self-report data for key outcome variables, including job-search activities, employment, and substance use during the extended follow-up. While the self-report measures used have been validated, additional means of collecting follow-up data are recommended. Fourth, the 9-month follow-up period was too short to provide sufficient data regarding job tenure. Future studies should consider using a 2-year follow-up period to allow more documentation of whether job tenure was clearly impacted. Finally, cost is a major concern about this type of intervention. An additional cost of $\$ 1,000$ in payments would almost double the cost of care per VR participant [3]. Further study is needed to determine (1) the relationship between the total cost of payments and outcome, (2) whether the payments result in secondary costs or savings in changes in other health service use, and (3) whether funding payments out of employer contracts is feasible and effective.

\section{CONCLUSIONS}

Overall, these findings are promising and suggest that the addition of CM techniques to VR programs increases participants' efforts to find employment and their resulting rate of entry into employment. In one sense, this finding should not be surprising. Employers and economists have developed a wide variety of payment incentives to shape employees' behavior. What is surprising is that clinicians working in VR programs, who focus on helping adults with disabilities return to work, apparently fail to recognize the potential of using financial incentives directly tied to the achievement of clinical goals to enhance their clinical programs. Like employers, many VR programs use financial incentives like pay and bonuses to reward work performance [24], but little evidence exists that they have applied these same incentives to directly reward job acquisition. While the current study directly supports the addition of CM interventions to enhance VR outcomes, it also suggests that VR programs should consider restructuring existing financial payments to include explicit links to these clinical goals.
In summary, this study demonstrates that adding a $\mathrm{CM}$ intervention to a VR program can positively affect program outcomes for dually diagnosed participants, increasing their overall rate of employment and jobsearch intensity. Further study must address the amount and structure of the reinforcement schedule, the generalizability to the broader population of VR participants, the effectiveness of adding CM interventions to other VR models such as supported employment and interventions like motivational interviewing, and the long-term effect of payments on VR participants' job tenure and sobriety.

\section{ACKNOWLEDGMENTS}

We thank Marylee Losardo, who assisted with subject recruitment, and John Clary, who assisted with clerical support.

Dr. Penk is now with Texas A\&M Health Science Center College of Medicine, College Station, Texas, and Dr. Hebert is now with Framingham State College, Framingham, Massachusetts.

This material was based on work supported by the VA Rehabilitation Research and Development Service (grant D2944R) and with resources of the New England Mental Illness Research, Education, and Clinical Center.

The authors have declared that no competing interests exist.

\section{REFERENCES}

1. Xie H, Dain BJ, Becker DR, Drake RE. Job tenure among persons with severe mental illness. Rehabil Couns Bull. 1997;40(4):230-39.

2. Resnick SG, Medak S, Baldino R, Corwel L, Rosenheck R. Eight progress reports on the Compensated Work Therapy (CWT)/Veterans Industries (VI) program. West Haven (CT): Department of Veterans Affairs Northeast Program Evaluation Center; 2004.

3. Blow FC, Gillon L, Dornfeld M. Comprehensive national evaluation of VHA Compensated Work Therapy. Ann Arbor (MI): Serious Mental Illness Treatment Research and Evaluation Center; 2001.

4. Hebert M, Drebing CE, Mueller L, Van Ormer EA. Job search decision making among vocational rehabilitation participants: A behavioral economics perspective. Am J Psychiatr Rehabil. 9:191-218. 
5. Rosenheck R. Fiscal year 2000 draft tables CWT progress report. West Haven (CT): Department of Veterans Affairs Northeast Program Evaluation Center; 2001.

6. Noble JH, Honberg RS, Hall LL, Flynn LM. A legacy of failure: The inability of the federal-state vocational rehabilitation system to serve people with severe mental illnesses. Arlington (VA): National Alliance for the Mentally Ill; 1997.

7. Drebing CE, Penk W, Rosenheck R. Readiness for competitive employment: Predicting "success" of CWT graduates. Presented at Annual Convention of the American Psychological Association; 2000 Aug 4-8; Washington, DC.

8. Drew D, Drebing CE, Van Ormer EA, Losardo M, Krebs C, Penk W, Rosenheck RA. Effects of disability compensation on participation in and outcomes of vocational rehabilitation. Psychiatr Serv. 2001;52(11):1479-84.

[PMID: 11684743]

9. Rosenheck R, Massari L, Astrachan B, Suchinsky R. Mentally ill chemical abusers discharged from VA inpatient treatment: 1976-1988. Psychiatr Q. 1990;61(4):237-49. [PMID: 2103008$]$

10. Drake RE, Brunett MF, Mueser KT. Substance use disorder and social functioning. In: Mueser KT, Tarrier N, editors. Schizophrenia in handbook of social functioning in schizophrenia. Needham Heights (MA): Allyn and Bacon; 1998.

11. Drake RE, Mueser KT. Psychosocial approaches to dual diagnosis. Schizophr Bull. 2000;26(1):105-18.

[PMID: 10755672]

12. Drake RE, McHugo GJ, Becker DR, Anthony WA, Clark RE. The New Hampshire study of supported employment for people with severe mental illness. J Consult Clin Psychol. 1996;64(2):391-99. [PMID: 8871423]

13. Acierno R, Donohue B, Kogan E. Psychological interventions for drug abuse: A critique and summation of controlled studies. Clin Psychol Rev. 1994;14(5):417-42.

14. Petry NM. A comprehensive guide to the application of contingency management procedures in clinical settings. Drug Alcohol Depend. 2000;58(1-2):9-25. [PMID: 10669051$]$

15. Higgins ST, Budney AJ, Bickel WK, Foerg FE, Donham R, Badger GJ. Incentives improve outcome in outpatient behavioral treatment of cocaine dependence. Arch Gen Psychiatry. 1994;51(7):568-76. [PMID: 8031230]

16. Stitzer ML, Bickel WK, Bigelow GE, Liebson IA. Effects of methadone dose contingencies on urinalysis test results of polydrug-abusing methadone-maintenance patients. Drug Alcohol Depend. 1986;18(4):341-48. [PMID: 3816530]

17. Budney AJ, Higgins ST, Radonovich KJ, Novy PL. Adding voucher-based incentives to coping skills and motivational enhancement improves outcomes during treatment for marijuana dependence. J Consult Clin Psychol. 2000;68(6): 1051-61. [PMID: 11142539]
18. Petry NM, Martin B, Cooney JL, Kranzler HR. Give them prizes, and they will come: Contingency management for treatment of alcohol dependence. J Consult Clin Psychol. 2000;68(2):250-57. [PMID: 10780125]

19. Silverman K, Svikis D, Robles E, Stitzer ML, Bigelow GE. A reinforcement-based therapeutic workplace for the treatment of drug abuse: Six-month abstinence outcomes. Exp Clin Psychopharmacol. 2001;9(1):14-23. [PMID: 11519628]

20. Milby JB, Schumacher JE, Raczynski JM, Caldwell E, Engle M, Michael M, Carr J. Sufficient conditions for effective treatment of substance abusing homeless persons. Drug Alcohol Depend. 1996;43(1-2):39-47. [PMID: 8957141]

21. Silverman K, Svikis D, Wong CJ, Hampton J, Stitzer ML, Bigelow GE. A reinforcement-based therapeutic workplace for the treatment of drug abuse: Three-year abstinence outcomes. Exp Clin Psychopharmacol. 2002;10(3):228-40. [PMID: 12233983]

22. Silverman K, Chatuape MA, Bigelow GE, Stitzer ML. Voucher-based reinforcement of attendance by unemployed methadone patients in a job skills training program. Drug Alcohol Depend. 1996;41(3):197-207. [PMID: 8842632]

23. Petry NM, Tedford J, Martin B. Reinforcing compliance with non-drug-related activities. J Subst Abuse Treat. 2001; 20(1):33-44. [PMID: 11239726]

24. Drebing CE, Van Ormer EA, Krebs C, Rosenheck R, Rounsaville B, Herz L, Penk W. The impact of enhanced incentives on vocational rehabilitation outcomes for dually diagnosed veterans. J Appl Behav Anal. 2005;38(3):359-72. [PMID: 16270845]

25. Vinokur A, Schul Y. The web of coping resources and pathways to reemployment following a job loss. J Occup Health Psychol. 2002;7(1):68-83. [PMID: 11827235]

26. National Institute on Drug Abuse [homepage on the Internet]. Washington (DC): National Institutes of Health; c2007 [updated 2005 Jan 21; cited 2007 Sep 14]. Budney AJ, Higgins ST. A community reinforcement approach: Treating cocaine addiction [about 15 screens]. Available from: http:// www.drugabuse.gov/TXManuals/CRA/CRA1a.html

27. Drebing CE, Losardo M, Van Ormer EA, Krebs C, Penk W, Nasser E, Ray H, Seibyl C, Rosenheck R. Vocational rehabilitation and older adults: Patterns in participation and outcome. J Rehabil. 2002;68(3):24-32.

28. MOW International Research Team. The meaning of work: An international view. London (England): Academic Press; 1987.

29. First MB. User's guide for the structured clinical interview for DSM-IV axis I disorders: SCID-I. New York (NY): Biometrics Research Department, New York Psychiatric Institute; 1996.

30. McHorney CA, Ware JE Jr, Lu JF, Sherbourne CD. The MOS 36-item Short-Form Health Survey (SF-36): III. Tests of data quality, scaling assumptions, and reliability across 
diverse patient groups. Med Care. 1994;32(1):40-66. [PMID: 8277801]

31. Vuori J, Vesalainen J. Labour market interventions as predictors of reemployment, job seeking activity and psychological distress among the unemployed. J Occup Org Psychol. 1999;72(4):523-38.

32. Bond GR, Becker DR, Drake RE, Vogler KM. A fidelity scale for the individual placement and support model of supported employment. Rehabil Couns Bull. 1997;40(4): 265-84.

33. Drake RE. A brief history of the individual placement and support model. Psychiatr Rehabil J. 1998;22(1):3-7.

34. Seibyl CL, Baldino R, Corwel L, Medak S, Rosenheck R. Final progress report on the Compensated Work Therapy (CWT)/Veterans Industries (VI) programs: Fiscal year 2001. West Haven (CT): Department of Veterans Affairs Northeast Program Evaluation Center; 2002.

35. Kashner TM, Rosenheck R, Campinell AB, Suris A, Crandall R, Garfield NJ, Lapuc P, Pyrcz K, Soyka T, Wicker A. Impact of work on health status among homeless, substance- dependent veterans: A randomized controlled trial. Arch Gen Psychiatry. 2002;59(10):938-44. [PMID: 12365881]

36. Roche Diagnostics Inc. Ontrak testcup 5 collection/urinalysis panel (07 64817). Indianapolis (IN): Roche Diagnostics Inc; 1998.

37. Roche Diagnostics Inc. Alcohol onsite assay for the qualitative detection of alcohol in urine and saliva (07 64108). Indianapolis (IN): Roche Diagnostics Inc; 1998.

38. Carney MA, Tennen H, Affleck G, Del Boca FK, Kranzler HR. Levels and patterns of alcohol consumption using timeline follow-back, daily diaries and real-time "electronic interviews.” J Stud Alcohol. 1998;59(4):447-54. [PMID: 9647427]

39. Cohen J. Statistical power analysis for the behavioral sciences. Hillsdale (NJ): Lawrence Erlbaum Associates, Inc; 1988.

Submitted for publication September 29, 2006. Accepted in revised form February 28, 2007. 